\title{
Simulation of heat diffusion in multi-layered construction systems for active IRT data analysis
}

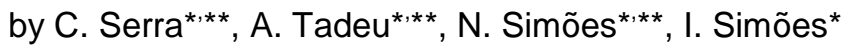 \\ * CICC, Department of Civil Engineering, University of Coimbra, Rua Luís Reis Santos - Pólo II, 3030-788 \\ Coimbra, Portugal, \{cserra, tadeu, nasimoes, mivsimoes\}@itecons.uc.pt \\ ** ITeCons, Rua Pedro Hispano s/n., 3030-289 Coimbra, Portugal
}

\begin{abstract}
In this paper, analytical solutions to simulate heat diffusion in layered media are presented. A phase-contrast approach to heat transfer results is taken, aiming at contributing to the interpretation of thermographic data obtained in building envelope inspections. Analytical phase-contrast results are computed in the frequency domain for case studies with varying material properties and thicknesses. An active IRT experimental campaign is performed to a test specimen and phase contrast calculations using analytical solutions for multi-layered systems are performed looking to simulate the experimental results. Heat transfer simulation results and IRT experimental results obtained in this study are compared with the intent to contribute to the understanding of active IRT tests results and to the definition of test parameters, when applied to construction elements which follow a multi-layered configuration.
\end{abstract}

\section{Introduction}

Since heat and moisture diffusion is sensitive to the presence of defects, the analysis of thermal pattern images collected via infrared thermography (IRT) has proven to be a useful non-destructive evaluation technique used for defect detection in a wide range of applications and sectors, including civil engineering ([1],[2]). Thermal imaging is mostly used in building diagnostics for detecting heat loss, missing or damaged insulation, thermal bridges, air leakages and excess moisture [3], using a passive approach. In passive IRT studies the materials and structures are tested in their natural state, usually considering steady-state conditions. However, by taking an active approach using an additional known heat source to generate the temperature difference which is not produced otherwise [4] a quantitative characterization of subsurface defects properties can be achieved by solving heat transfer problems using active IRT data results.

In active IRT different types of thermal stimulation are used to produce a temperature gradient in the test specimen and various data analysis methods are used to acquire results recorded in a transient regime. Maldague and Marinetti [6] introduced the pulse-phase thermography (PPT) processing technique in which test specimens are pulse-heated but thermal waves are unscrambled by performing the Fourier transform of the temperature decay, enabling the computation of phase images. Analysis in PPT is performed in transient regime giving information in both the time domain and the frequency domain. Arndt [7] concluded that PPT is an ideal approach for IRT use in Civil Engineering applications, since phase images allow for deeper probing compared to thermal images, and thus enhanced defect detectability, as well as enhanced resolution of defect geometry. Also, phase images are less influenced by surface characteristics and less sensitive to non-uniform heating. Furthermore, in PPT there is no need for previous knowledge of the position of nondefect areas which is necessary in simple thermal contrast processing. Maldague [5] stated that comparisons between heat transfer simulation models and experimental results, although not straightforward, can give insight into the general thermal behaviour of the sample being studied, and may be useful to establish the limits of the effectiveness of IRT and to set inspection parameters. The present study aims to contribute to the interpretation of IRT tests results performed on building elements which in great part follow a multi-layered configuration. Focused on studying the effects that changing the configuration of a layered system has on IRT experimental studies, a comparative study between the simulation of heat diffusion in a multi-layered system and active IRT test results is carried out.

As mentioned, in this work heat diffusion is studied considering building elements as multi-layered systems. Heat transfer studies in multi-layered systems may be performed based on Green's analytical functions [8],[9]. In order to compute phase-contrast results in the frequency domain the analytical expressions proposed by Tadeu and Simões [10], incorporating Green's functions for calculating transient heat transfer by conduction in a multi-layer system subject to a planar heat source, are used. An application to a number of case studies is performed by changing the thermal properties of the materials and thicknesses of the layers. Active IRT experimental tests are performed to a particular test specimen and the resulting phase-contrast curves are confronted with the simulation results. It is expected that the results of the comparative study contribute to the interpretation of quantitative data results from IRT test performed on building envelopes. 


\section{Methods}

As stated, the quantitative interpretation of thermographic data requires solving heat transfer problems. In this study, heat diffusion in layered media in transient regime is simulated using the analytical solutions described in the following section and the effect that the presence of a certain layer has on the heat waves propagating within a system of $m$ layers is studied using a Phase-contrast approach. In order to study the influence of a given layer on phase-contrast results, the phase of the thermal waves is computed for a system with a number of $m$ layers and for a system with $m-1$ layers (i.e. without the presence of the layer being studied). The difference between both gives the phase-contrast results for any frequency.

In the following section an analytical study is performed in order to show the effect that changing the number of layers, thermal properties and the thickness of each layer has on phase-contrast curves. After, active IRT experimental tests are presented and compared with the analytical modelling results for a case study of a specimen following a multilayered configuration. The active thermography measurements were performed in a laboratorial space with a video recording during the specimens surface stimulation (in reflection mode) from two halogen lamps (2500 W each) with a rectangular heating curve (square pulse), using $100 \%$ of the available heating power, followed by a cooling down process.

\section{Simulation of heat diffusion in a multi-layered system using analytical solutions}

\subsection{Problem definition}

Thermal wave phase results are computed for a system of $m$ plane layers of infinite extent in the $x$ and $z$ directions, bounded by a flat semi-infinite medium $M_{0}$ at the top (see Figure 1 ). The system is subjected to a temperature $t_{o b}$ at the bottom external surface, at interface $m+1$.

For each layer the thermal properties and thickness $h$ may differ.

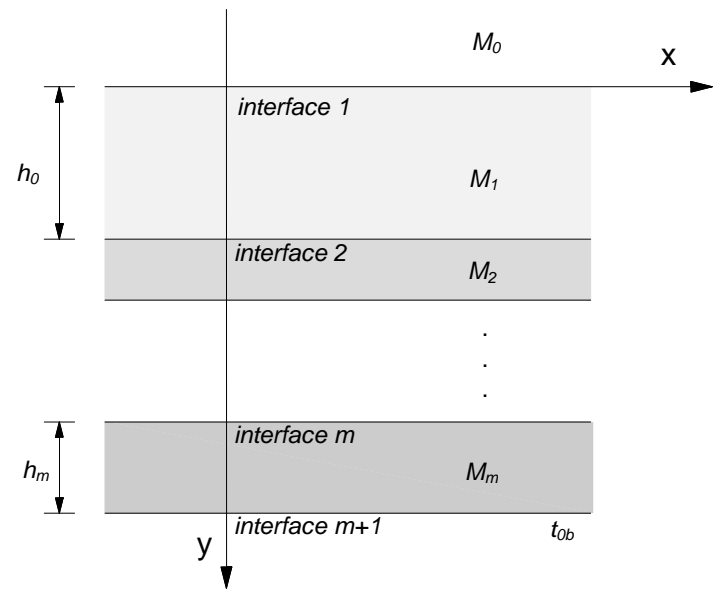

Fig. 1. Geometry of the problem of heat diffusion generated by a point heat source in a multi-layered system.

A planar heat source is placed somewhere in the domain and the resulting heat field is computed by adding the heat source terms, equal to those in an unbounded space, with the set of surface terms which are generated at each interface between layers to ensure boundary conditions at each interface. The analytical solutions are briefly explained in the following paragraphs. A full description of these equations can be found in [11], where the 2.5D solution for multilayered systems has been proposed and validated.

\subsection{Problem Formulation}

The transient heat transfer by conduction in each layer is expressed by:

$$
\lambda_{j}\left(\frac{\partial^{2}}{\partial y^{2}}\right) T(t, y)=\rho_{j} c_{j} \frac{\partial T(t, y)}{\partial t}
$$

in which $t$ is time, $T(t, y)$ is temperature, the subscript $j$ identifies the layer, $\lambda$ is the thermal conductivity, $\rho$ is density and $c$ is specific heat. These thermal properties are constant in each layer and do not change from point to point. By applying a Fourier transform in the time domain:

$$
\left(\frac{\partial^{2}}{\partial y^{2}}+\left(\sqrt{\frac{-\mathrm{i} \omega}{\alpha_{j}}}\right)^{2}\right) \hat{T}(\omega, y)=0,
$$


where $\mathrm{i}=\sqrt{-1}, \alpha_{j}=\lambda_{j} /\left(\rho_{j} c_{j}\right)$ is the thermal diffusivity of the layer $j$, and $\omega$ is the frequency.

The total heat field is found by adding the surface terms which arise within each layer and at each interface, in order to satisfy the boundary conditions at the interfaces, i.e. continuity of temperatures and normal flows between layers. For layer $j$, the heat surface terms at the upper and lower interfaces are expressed by:

$$
\begin{aligned}
& \tilde{T}_{j 1}(\omega, y)=E_{0 j} \frac{E_{j 1}}{v_{0 j}} A_{0 j}^{t}, \\
& \tilde{T}_{j 2}(\omega, y)=E_{0 j} \frac{E_{j 2}}{v_{0 j}} A_{0 j}^{b},
\end{aligned}
$$

where $E_{0 j}=\frac{1}{\lambda_{j}}, E_{j 1}=\mathrm{e}^{-\mathrm{i} v_{0 j}\left|y-\sum_{i=1}^{j-1} h_{l}\right|}, E_{j 2}=\mathrm{e}^{-\mathrm{i} v_{0 j}\left|y-\sum_{i=1}^{j} h_{l}\right|}, v_{0 j}=\sqrt{-\mathrm{i} \omega / \alpha_{j}}, h_{l}$ is the thickness of the layer $I$ and $A_{0 j}^{t}$ and $A_{0 j}^{b}$ are a priori unknown potential amplitudes. A system of $2(m+1)$ equations is derived, by ensuring the continuity of temperatures and heat flows along the $m$ interfaces between layers, and by imposing the temperature $\hat{t}_{0 b}$ at the bottom external surface. $\hat{t}_{0 b}$ is obtained by Fourier transformation of $t_{0 b}$ in the time domain. All the terms are organized according to the form:

$$
\left[\begin{array}{cccccc}
-1 & -1 & \mathrm{e}^{-\mathrm{i} v_{01} h_{1}} & \ldots & 0 & 0 \\
\frac{1}{\lambda_{0} v_{00}} & -\frac{1}{\lambda_{1} v_{01}} & -\frac{\mathrm{e}^{-\mathrm{i} v_{01} h_{1}}}{\lambda_{1} v_{01}} & \ldots & 0 & 0 \\
0 & \mathrm{e}^{-\mathrm{i} v_{01} h_{1}} & -1 & \ldots & 0 & 0 \\
0 & -\frac{\mathrm{e}^{-i v_{n 1} h_{1}}}{\lambda_{1} v_{01}} & \frac{1}{\lambda_{1} v_{01}} & \ldots & 0 & 0 \\
\ldots & \ldots & \ldots & \ldots & \ldots & \ldots \\
0 & 0 & 0 & \ldots & -1 & \mathrm{e}^{-\mathrm{i} v_{0 m} h_{m}} \\
0 & 0 & 0 & \ldots & -\frac{1}{\lambda_{1} v_{0 m}} & -\frac{\mathrm{e}^{-i v_{0 m} h_{m}}}{\lambda_{m} v_{0 m}} \\
0 & 0 & 0 & \ldots & \frac{\mathrm{e}^{-\mathrm{i} v_{n m} h_{m}}}{\lambda_{m} v_{0 m}} & \frac{1}{\lambda_{m} v_{0 m}}
\end{array}\right]\left[\begin{array}{c}
A_{00}^{b} \\
A_{01}^{t} \\
A_{01}^{b} \\
\ldots \\
A_{0 m}^{t} \\
A_{0 m}^{b}
\end{array}\right]=\left[\begin{array}{c}
0 \\
0 \\
0 \\
\ldots \\
0 \\
\hat{t}_{0 b}
\end{array}\right] .
$$

Solving this system gives the amplitude of the surface terms at each interface, leading to the following temperature and heat flux fields at layer $j$ :

$$
\tilde{T}(\omega, y)=E_{0 j}\left(\frac{E_{j 1}}{v_{0 j}} A_{0 j}^{t}+\frac{E_{j 2}}{v_{0 j}} A_{0 j}^{b}\right) \text { if } \sum_{l=1}^{j-1} h_{l}<y<\sum_{l=1}^{j} h_{l} .
$$

Thermal wave phase results are then computed directly in the frequency domain. The thermal wave phase is the arctangent of the quotient of the imaginary part of the frequency domain temperature response divided by the real part.

\subsection{Application}

\subsubsection{Case studies}

The effect produced by changing the number of layers, the thermal properties and the thickness of the layers in the thermal wave phase calculations was investigated by introducing such changes in several case studies. Figure 2 illustrates the geometrical configuration of three of the cases studied:

- Case I (see Figure 2.a)) is a simple two layered system of two different materials; layer $M_{1}$ is the layer under study; a receiver is placed in layer $M_{2}$ at a distance $d$ away from the interface between the two layers. $d$ is changed in order to study the influence of changing the depth at which the layer $M_{1}$ is located.

- Case II (see Figure 2.b)) is a system of four layers; layer $M_{1}$ is under study; the receiver is placed at the interface between layers $M_{2}$ and $M_{3}$; layer $M_{2}$ has variable thickness $d$ to study the effect of changing the depth at which the material in layer $M_{1}$ is placed; layer $M_{3}$ has a thickness of 0,003 $\mathrm{m}$ and simulates surface thermal resistance; $M_{4}$ is a layer of air.

- Case III (see Figure 2.c)) is a system of seven layers simulating a multi-layered building element consisting of a core material (layer $M_{4}$ ) and a coating material on each side (layers $M_{3}$ and $M_{5}$ ). Layer $M_{4}$ is under study; layers $M_{2}$ and $M_{6}$ represent the surface thermal resistance; $M_{1}$ and $M_{7}$ are layers of air. 


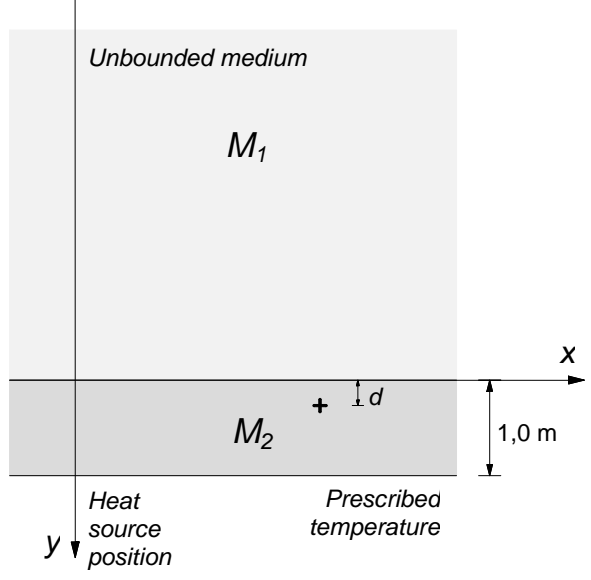

a)

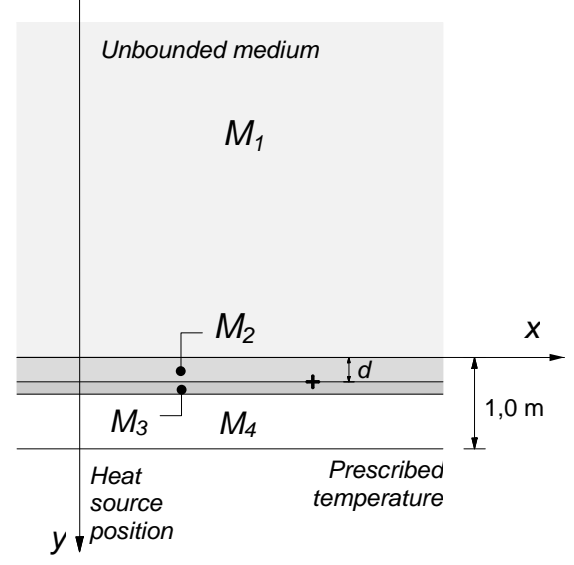

b)

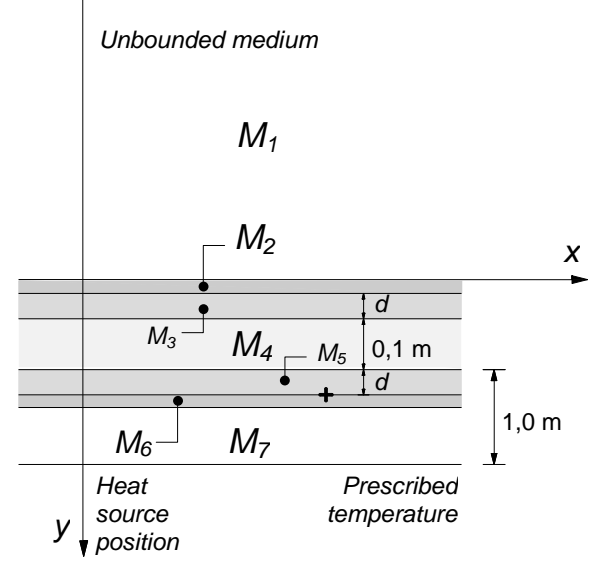

c)

Fig. 2. Geometries of the case studies: a) Case I; b) Case II; c) Case III.

In all cases the thermal properties of the layer being investigated were changed according to Table 1.

Table 1. Different material properties considered for the layer under study.

\begin{tabular}{|c|c|c|c|}
\hline Material & $\begin{array}{c}\text { Conductivity } \\
\lambda \\
\left(\mathrm{W} /\left(\mathrm{m} \cdot{ }^{\circ} \mathrm{C}\right)\right)\end{array}$ & $\begin{array}{c}\text { Specific } \\
\text { heat } c \\
\left(\mathrm{~J} / \mathrm{Kg} \cdot{ }^{\circ} \mathrm{C}\right)\end{array}$ & $\begin{array}{c}\text { Density } \\
\rho \\
\left(\mathrm{Kg} / \mathrm{m}^{3}\right)\end{array}$ \\
\hline ICB & 0,038 & 1560 & 100 \\
\hline MDF & 0,12 & 1550 & 712 \\
\hline wood & 0,29 & 1340 & 900 \\
\hline gypsum board & 0,35 & 840 & 900 \\
\hline basalt & 1,6 & 840 & 2850 \\
\hline granite & 3 & 840 & 2600 \\
\hline steel & 55 & 460 & 7800 \\
\hline copper & 380 & 380 & 8900 \\
\hline
\end{tabular}

Additionally, in order to visualize the potential limits of the phase-contrast analysis, calculations were performed for a virtual material with very low conductivity (conductivity $\lambda=0,12 \times 10^{-4} \mathrm{~W} /\left(\mathrm{m} .{ }^{\circ} \mathrm{C}\right.$ ), specific heat $C=1550 \mathrm{~J} / \mathrm{Kg} .{ }^{\circ} \mathrm{C}$ and density $\left.\rho=712 \mathrm{Kg} / \mathrm{m}^{3}\right)$ and a material with very high conductivity $\left(\lambda=380 \times 10^{4} \mathrm{~W} /\left(\mathrm{m} .{ }^{\circ} \mathrm{C}\right), c=380 \mathrm{~J} / \mathrm{Kg} .{ }^{\circ} \mathrm{C}\right.$ and $\rho=8900$ $\mathrm{Kg} / \mathrm{m}^{3}$ ). In all calculations the layers $M_{2}$, in cases I and II, and layers $M_{3}$ and $M_{5}$, in Case III, have the thermal properties of mortar $\left(\lambda=1,4 \mathrm{~W} /\left(\mathrm{m} .{ }^{\circ} \mathrm{C}\right), c=880 \mathrm{~J} / \mathrm{Kg} .{ }^{\circ} \mathrm{C}\right.$ and $\left.\rho=2300 \mathrm{Kg} / \mathrm{m}^{3}\right)$. The air layers have $\lambda=0,026 \mathrm{~W} /\left(\mathrm{m} .{ }^{\circ} \mathrm{C}\right), c=1000 \mathrm{~J} / \mathrm{Kg} .{ }^{\circ} \mathrm{C}$ and $\rho=1,293 \mathrm{Kg} / \mathrm{m}^{3}$. For each case study the distance/thickness $d$ was changed $(d=0,02 \mathrm{~m}$ and $d=0,04 \mathrm{~m})$ in order to simulate a change in depth of the layer under study.

\subsubsection{Phase-contrast results}

For each one of the configurations shown, calculations were made for the layer under study with each of the material properties given in table 1, located at a depth of $d=0,02 \mathrm{~m}$ and $d=0,04 \mathrm{~m}$. The remaining layers have constant material properties. Phase-contrast is obtained by computing the difference between phase calculations for the layer under study with the thermal properties shown in Table 1 and phase calculations for the layer under study with the thermal properties of mortar. The following graphs (see Figure 3, 4 and 5) show the phase-contrast results obtained. The resulting curves show that phase-contrast is negative for materials with lower conductivity than mortar (ICB, MDF, wood, gypsum board) and is positive for materials with conductivity higher than that of mortar (basalt, granite, steel, copper). The curves show a peak in the frequency spectra at maximum phase-contrast $\Delta \Phi_{\max }$, which corresponds to the characteristic frequency $f_{c h}$ used in defect detection and characterization experimental IRT studies (as seen in figure 5.a)). The defect detection threshold is given by the blind frequency $f_{b}$ at zero phase-contrast. In all cases the change in depth from $0,02 \mathrm{~m}$ to $0,04 \mathrm{~m}$ resulted in a decrease in the characteristic frequency $f_{c h}$ and blind frequency $f_{b}$ values, and in a decrease in the $\Delta \Phi_{\max }$ value. 


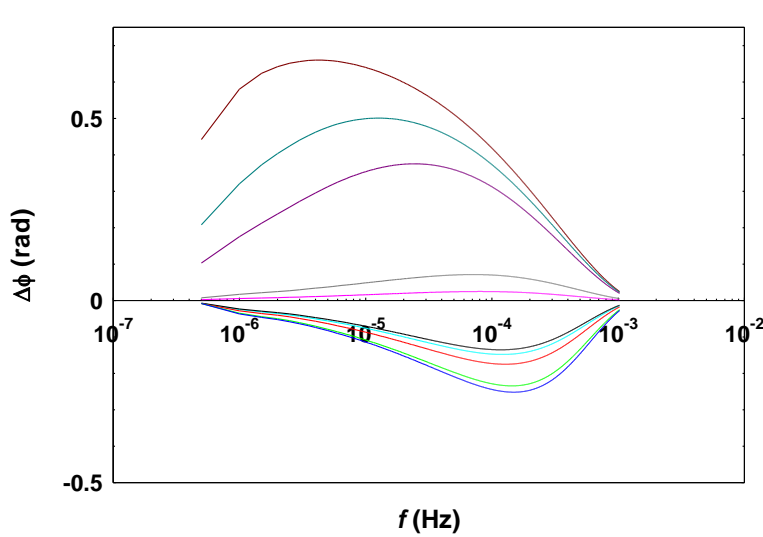

a)

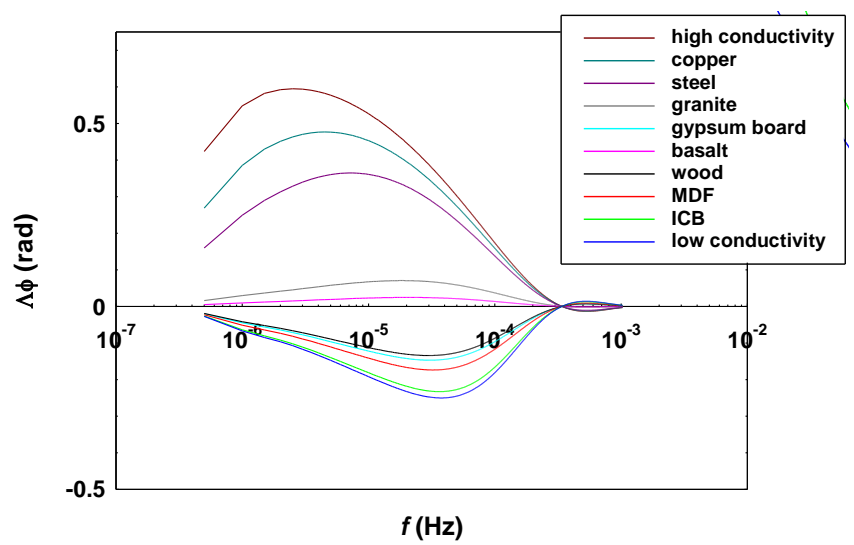

b)

Fig. 3. Phase-contrast results for Case l: a) receiver placed at $d=0,02 \mathrm{~m}$; b) receiver placed at $d=0,04 \mathrm{~m}$.

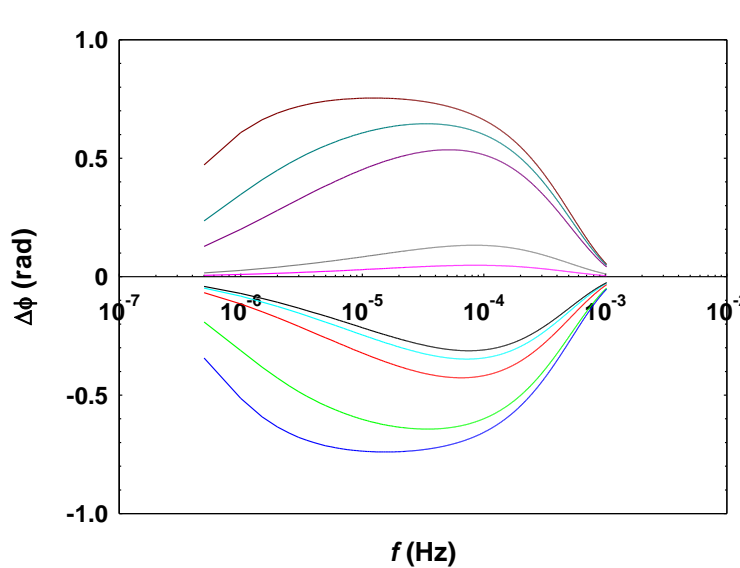

a)

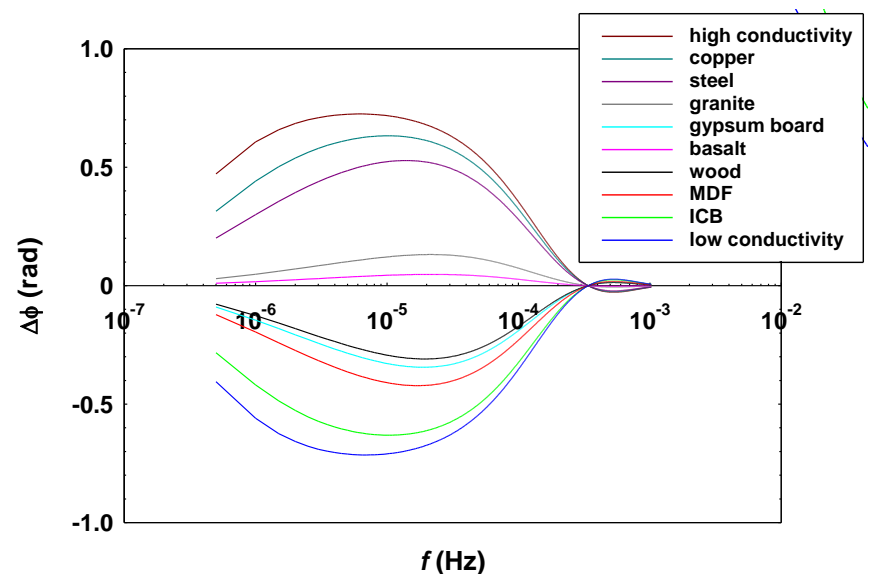

b)

Fig. 4. Phase-contrast results for Case II: a) layer $M_{2}$ with thickness of $d=0,02 \mathrm{~m}$; b) layer $M_{2}$ with thickness of $d=0,04 \mathrm{~m}$.

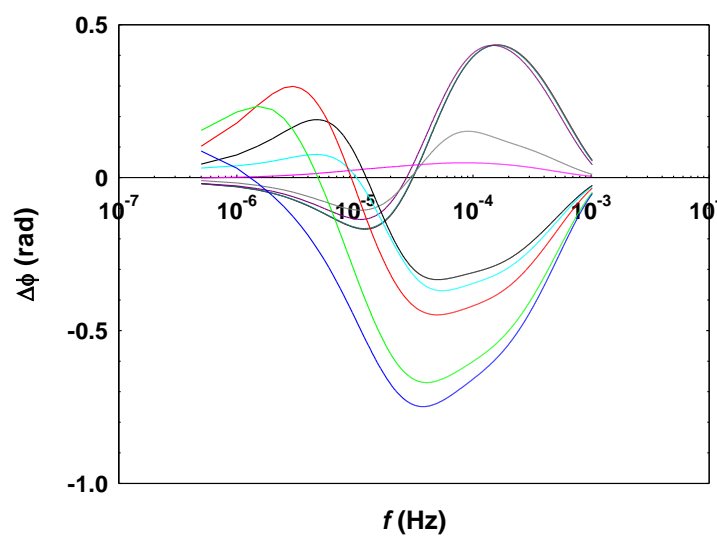

a)

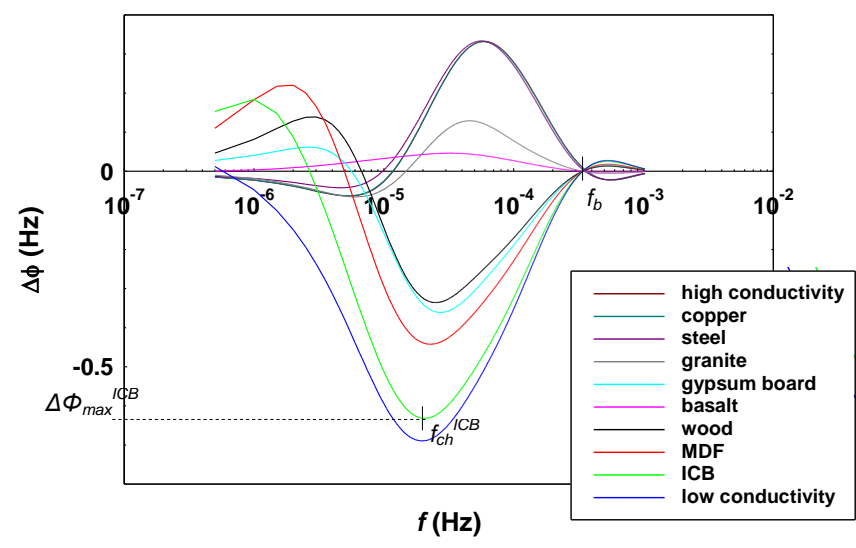

b)

Fig. 5. Phase-contrast results for Case III: a) layer $M_{4}$ located at a depth of $d=0,02 \mathrm{~m}$; b) layer $M_{4}$ located at a depth of $d=0,04 \mathrm{~m}$.

In particular for Case III, the peak in phase-contrast $\Delta \Phi_{\max }$ is clearly defined. The following table shows the maximum phase-contrast $\Delta \Phi_{\max }$ and the corresponding characteristic frequency $f_{c h}$ values obtained for each material considered in layer $M_{4}$, located at a depth of $d=0,02 \mathrm{~m}$ and $d=0,04 \mathrm{~m}$. 
Table 2. Case III phase-contrast results for the different material properties considered for layer $M_{4}$, located at a depth of $d=0,02 \mathrm{~m}$ and $d=0,04 \mathrm{~m}$.

\begin{tabular}{|c|c|c|c|c|}
\hline \multirow{2}{*}{ Material } & \multicolumn{2}{|c|}{$d=0,02 \mathrm{~m}$} & \multicolumn{2}{c|}{$d=0,04 \mathrm{~m}$} \\
\cline { 2 - 5 } & $\begin{array}{c}\text { maximum } \\
\text { phase-contrast } \\
\Delta \Phi_{\max }(\mathrm{rad})\end{array}$ & $\begin{array}{c}\text { characteristic } \\
\text { frequency } f_{c h} \\
(\mathrm{~Hz})\end{array}$ & $\begin{array}{c}\text { maximum } \\
\text { phase-contrast } \\
\Delta \Phi_{\max }(\mathrm{rad})\end{array}$ & $\begin{array}{c}\text { characteristic } \\
\text { frequency } f_{c h} \\
(\mathrm{~Hz})\end{array}$ \\
\hline low conductivity & $-0,749$ & $3,75 \times 10^{-5}$ & $-0,690$ & $1,95 \times 10^{-5}$ \\
\hline ICB & $-0,670$ & $4,05 \times 10^{-5}$ & $-0,632$ & $2,05 \times 10^{-5}$ \\
\hline MDF & $-0,449$ & $4,95 \times 10^{-5}$ & $-0,442$ & $2,30 \times 10^{-5}$ \\
\hline wood & $-0,333$ & $5,05 \times 10^{-5}$ & $-0,336$ & $2,50 \times 10^{-5}$ \\
\hline gypsum board & $-0,370$ & $5,50 \times 10^{-5}$ & $-0,361$ & $2,75 \times 10^{-5}$ \\
\hline basalt & 0,049 & $8,40 \times 10^{-5}$ & 0,046 & $3,20 \times 10^{-5}$ \\
\hline granite & 0,152 & $9,10 \times 10^{-5}$ & 0,129 & $4,60 \times 10^{-5}$ \\
\hline steel & 0,433 & $1,46 \times 10^{-4}$ & 0,333 & $5,65 \times 10^{-5}$ \\
\hline copper & 0,433 & $1,59 \times 10^{-4}$ & 0,331 & $5,90 \times 10^{-5}$ \\
\hline high conductivity & 0,434 & $1,60 \times 10^{-4}$ & 0,332 & $5,90 \times 10^{-5}$ \\
\hline
\end{tabular}

\section{Experimental study}

In this section a comparative study of experimental and numerical results using the described analytical model is presented. First, the experimental set up and test parameters used in the experimental studies are explained. Then phase-contrast computations for a multi-layered model simulating the test specimen are presented and a comparison of the obtained results is performed.

\subsection{Experimental Apparatus and test procedures}

Active IRT experimental tests were performed to a test specimen. In order to mimic a general multi-layered building element with changeable components, a glass box was filled with wet sand and an object inclusion was inserted in the box. The glass was covered with dark film in order to control emissivity and eliminate the reflectivity of the glass surface. The inserted object has changeable position and nature, however, in this paper only the results obtained for an extruded polystyrene foam thermal insulation (XPS) board defect are presented. An extensive experimental campaign, which is not covered in this paper, was performed to assess the influence of environmental parameters.

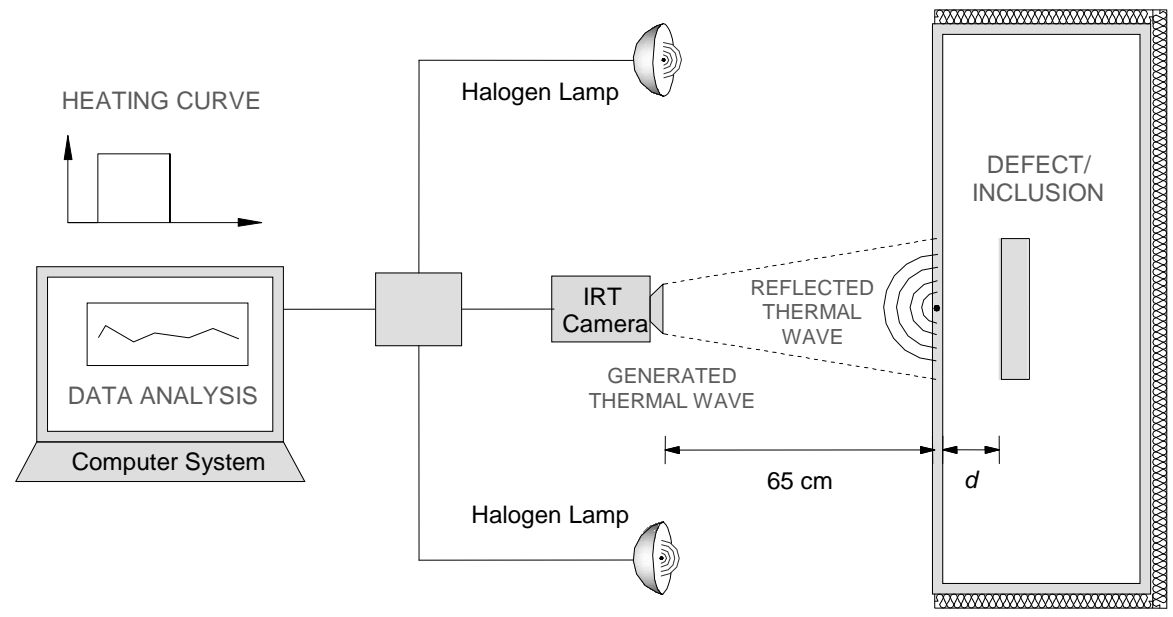

Fig. 6. Scheme of the experimental apparatus.

The tests were performed in a room with controlled temperature, humidity and lighting in order to minimize the influence of environmental parameters. The sand in the box was ensured to be saturated at the beginning of each test. The film covering the specimen surface has an emissivity of $\varepsilon=0,95$. The glass box was surrounded with insulation material. Two halogen lamps with a power of $2500 \mathrm{~W}$ each were placed $0,65 \mathrm{~m}$ away from the specimen surface (see Figure 6). After an initial period of 100 seconds, the surface was heated for 200 seconds, followed by a cooling down period which was recorded for 3796 seconds. For a total of 4096 seconds infrared images were recorded each 32 seconds, resulting in a frame rate of $0.03125 \mathrm{~Hz}$. The thermal pattern image captured in each frame by the IRT camera 
is $0,275 \times 0,212 \mathrm{~m}^{2}$. In each test 128 frames were recorded and stored in the computer connected to the camera (using the software IR NDT version 1.74 from Automation Technology).

\subsection{Case Study}

\subsubsection{Test Specimen}

The following image is a scheme of the test specimen built for the experimental campaign. A $0,2 \times 0,2 \mathrm{~m}^{2} \times P S$ board is centred in the image captured by the camera and its depth $d$ is changed throughout the several tests.

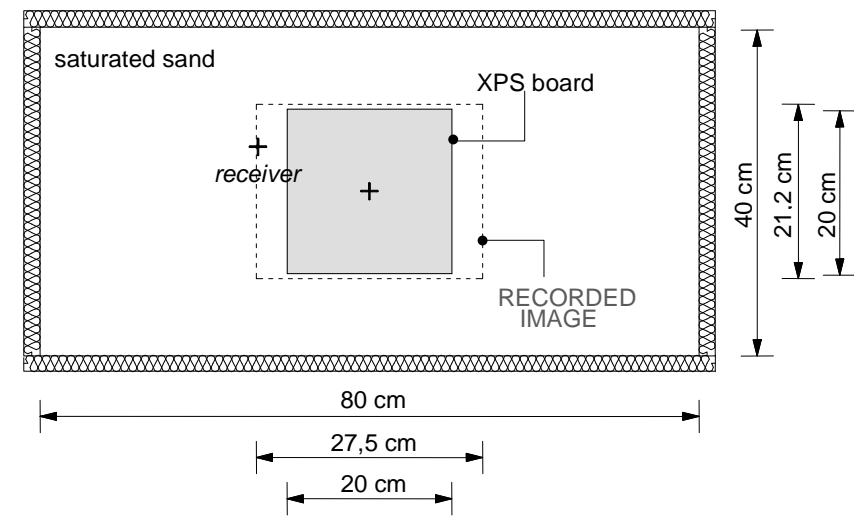

a)

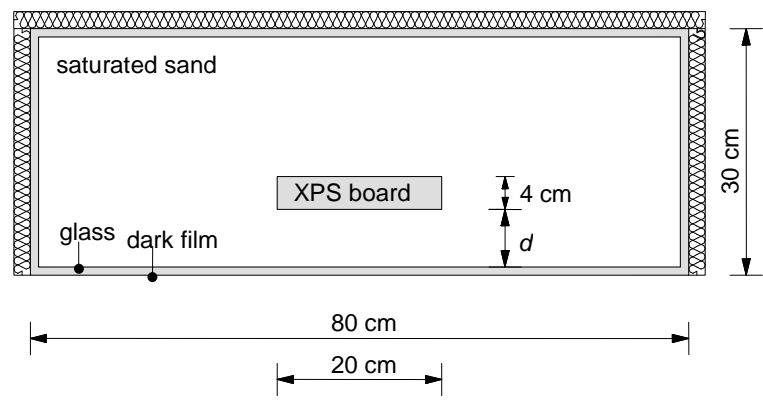

b)

Fig. 7. Scheme of the test specimen: a) front view; b) view from the top.

\subsubsection{Analytical model}

The image in Figure 8 illustrates the multi-layered system used to simulate analytically phase-contrast calculations. The case study is a nine layers system simulating the experimental case study. The XPS layer is the layer being studied, which corresponds to the defect/inclusion found in typical IRT defect characterization tests. It is surrounded by a layer of saturated sand on both sides. The layer of sand which is nearest to the surface has variable depth, simulating the buried depth of the XPS layer. The sand is confined by a 0,006 m thick glass layer. The Rsi layers simulate the surface thermal resistances. The heat source is located at the bottom.

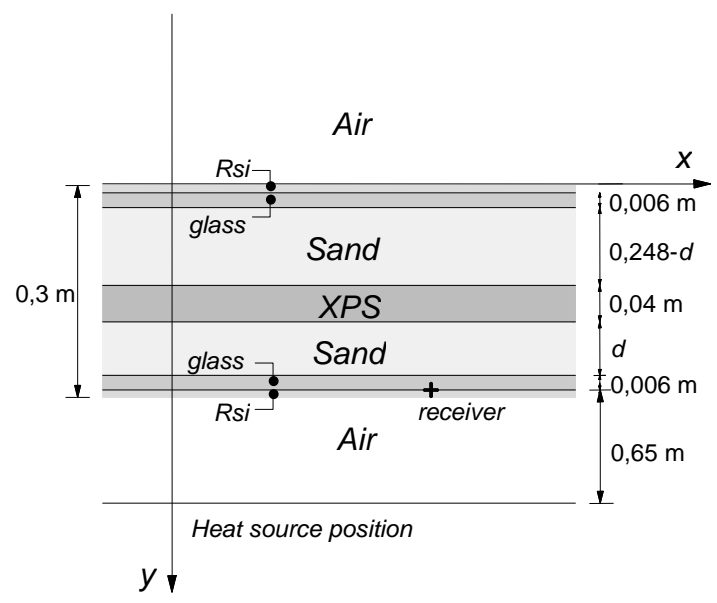

Fig. 8. Geometry of the multi-layer model of the test specimen.

Table 3. Material properties considered for the multi-layered model of the test specimen.

\begin{tabular}{|c|c|c|c|}
\hline Material & $\begin{array}{c}\text { Conductivity } \lambda \\
\left(\mathrm{W} /\left(\mathrm{m} .{ }^{\circ} \mathrm{C}\right)\right)\end{array}$ & $\begin{array}{c}\text { Specific heat } \\
\mathrm{C}\left(\mathrm{J} / \mathrm{Kg} .{ }^{\circ} \mathrm{C}\right)\end{array}$ & $\begin{array}{c}\text { Density } \rho \\
\left(\mathrm{Kg} / \mathrm{m}^{3}\right)\end{array}$ \\
\hline Glass & 1 & 750 & 2500 \\
\hline XPS & 0,033 & 1450 & 30 \\
\hline Saturated sand & 1,840 & 1000 & 1850 \\
\hline Air & 0,026 & 1000 & 1,293 \\
\hline
\end{tabular}


The thermal properties of the materials used in the analytical simulations were estimated using characterization tests, resulting in the values shown in table 3. Thermal conductivity was obtained using the Guarded Hot Plate method, according to EN 12667:2001, while the specific heat was determined using the ratio method.

\subsection{Results}

\subsubsection{Phase-contrast results}

Phase results for specific points of the recorded image were extracted (receivers). This was done by applying a fourier transform to the time domain temperature pattern results recorded. For a total observation time of 4096 seconds at a frame rate of $f=1 / 32=0,03125 \mathrm{~Hz}$, the resulting number of frequency steps is 256 and the frequency increment is $\Delta t=1,22 \times 10^{-4} \mathrm{~Hz}$. Then, phase-contrast results for specific receivers were computed by calculating the difference between the phase results measured at such receivers and the phase recorded at a receiver located outside the area of influence of the defect (see Figure 7.a)), which is no longer affected by the its inclusion.

The resulting phase-contrast curves for a receiver located at the centre of the image, where the amplitude of the phase is expected to be greater, are shown in the following graph (Figure 9), along with the maximum phase-contrast $\Delta \Phi_{\max }$ and corresponding characteristic $f_{c h}$ values obtained for the various depths considered:

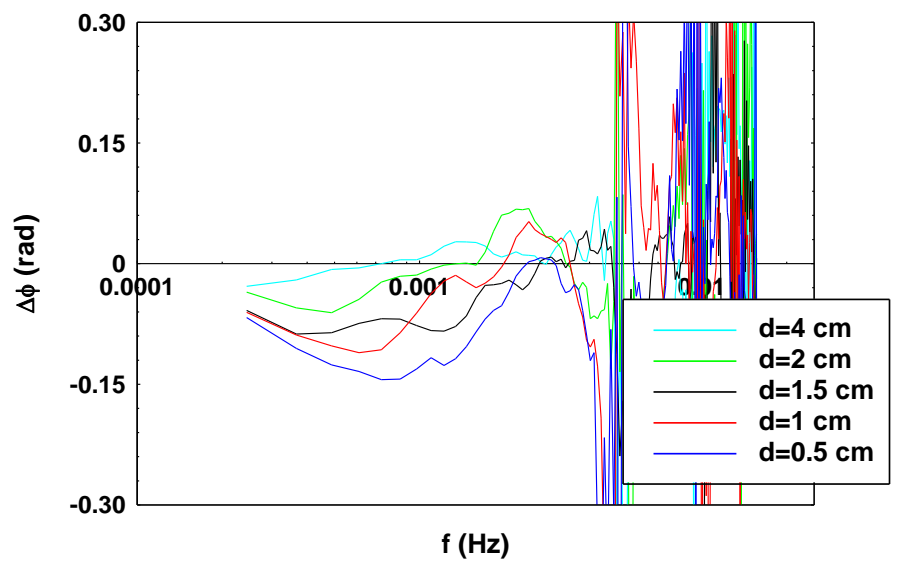

\begin{tabular}{|c|c|c|}
\hline$d(\mathrm{~cm})$ & $f_{c h}(\mathrm{~Hz})$ & $\Delta \Phi_{\max }(\mathrm{rad})$ \\
\hline 4 & $2,44 \times 10^{-4}$ & $-2,82 \times 10^{-2}$ \\
\hline 2 & $4,88 \times 10^{-4}$ & $-6,09 \times 10^{-2}$ \\
\hline 1,5 & $3,66 \times 10^{-4}$ & $-8,75 \times 10^{-2}$ \\
\hline 1 & $6,10 \times 10^{-4}$ & $-0,11060$ \\
\hline 0,5 & $7,32 \times 10^{-4}$ & $-0,14423$ \\
\hline
\end{tabular}

Fig. 9. Experimental phase-contrast results for a XPS inclusion located at various depths.

The graph in Figure 10 shows the phase-contrast results, for the same receiver located at the centre of the image, using the analytical heat diffusion multi-layered model of the specimen used in the previous experimental part of this study (see Figure 8). Results were computed using 256 frequency steps and a frequency increment of $\Delta f=1,22 \times 10^{-4}$ $\mathrm{Hz}$, and the thermal properties in Table 3.

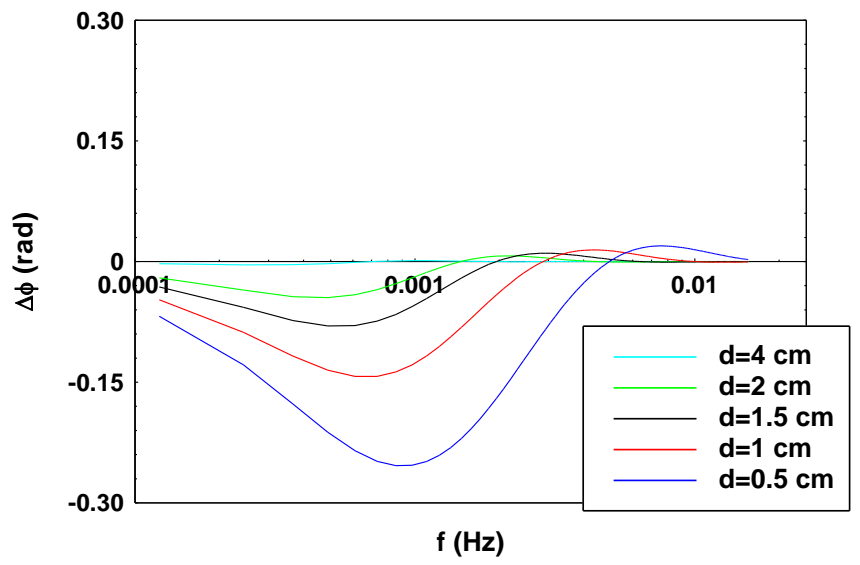

\begin{tabular}{|c|c|c|}
\hline$d(\mathrm{~cm})$ & $f_{c h}(\mathrm{~Hz})$ & $\Delta \Phi_{\max }(\mathrm{rad})$ \\
\hline 4 & $2,44 \times 10^{-4}$ & $-4,13 \times 10^{-3}$ \\
\hline 2 & $4,88 \times 10^{-4}$ & $-4,48 \times 10^{-2}$ \\
\hline 1,5 & $4,88 \times 10^{-4}$ & $-8,01 \times 10^{-2}$ \\
\hline 1 & $6,10 \times 10^{-4}$ & $-0,14275$ \\
\hline 0,5 & $8,54 \times 10^{-4}$ & $-0,2537$ \\
\hline
\end{tabular}

Fig. 10. Phase-contrast results for the heat diffusion simulation of an XPS inclusion located at various depths.

\subsubsection{Phase-contrast image results}

The following picture shows the phase-contrast images which correspond to the experimental results. The phase images are snapshots taken at the frequency of maximum phase contrast $\Delta \Phi_{\max }$, the characteristic frequency $f_{c h}$ and at the blind frequency $f_{b}$ which defines de detectability threshold, for an XPS board placed at the depths of $d=0,5 \mathrm{~cm}$, $d=1 \mathrm{~cm}$ and $d=2 \mathrm{~cm}$. 


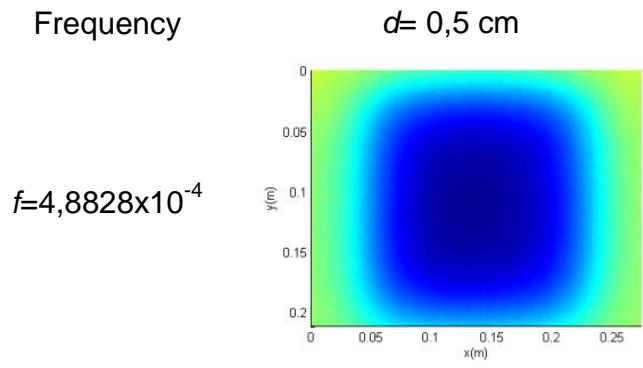

$t=6,1035 \times 10^{-4}$

$t=7,3242 \times 10^{-4}$

$t=1,4648 \times 10^{-3}$

$t=2,0752 \times 10^{-3}$

$t=2,5635 \times 10^{-3}$
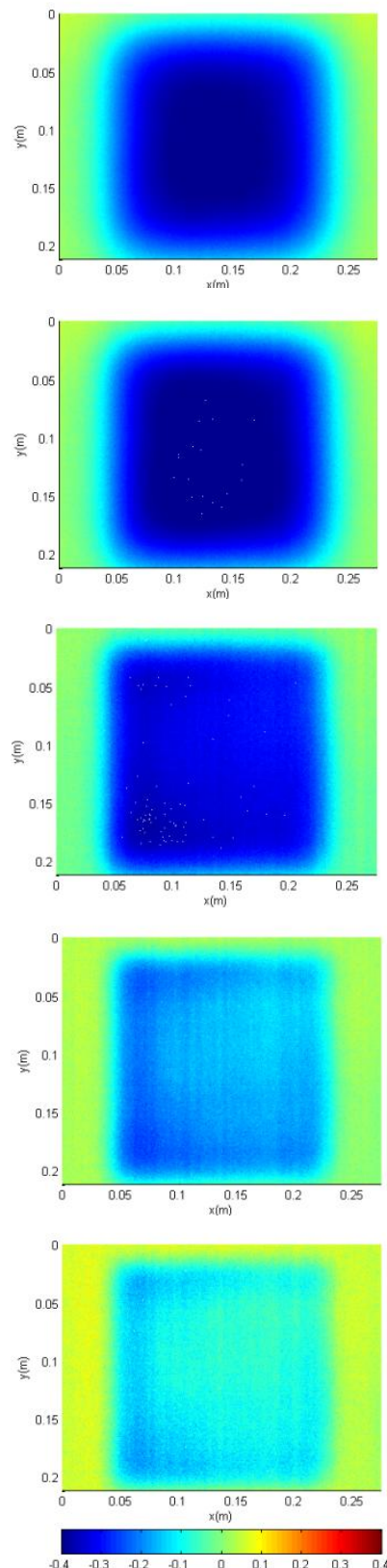

$d=1 \mathrm{~cm}$
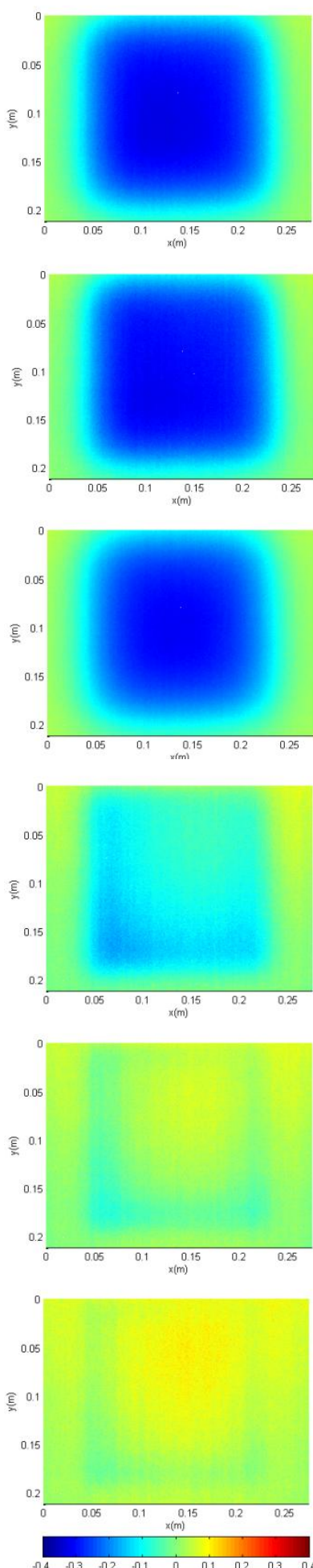

$d=2 \mathrm{~cm}$
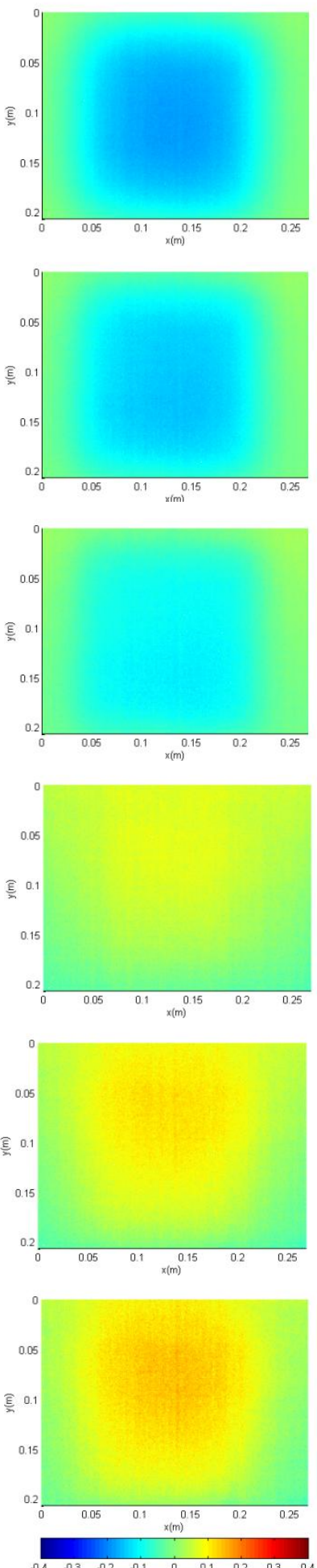

Fig. 11. Phase-contrast image experimental results for a test specimen with an XPS inclusion located at various depths.

\subsubsection{Discussion of results}

As predicted in the previous analytical study (see section 3 of this paper), the change in depth resulted, in each case, in a decrease in the $f_{c h}$ and $f_{b}$ values, as well as in a decrease in the $\Delta \Phi_{\max }$ value. This behaviour is can be seen both in the analytical (Figure 10) and the experimental results (Figure 9). For a depth of $4 \mathrm{~cm}$ the defect is no longer 
visible with a frequency increment of $\Delta f=1,22 \times 10-4 \mathrm{~Hz}$. In this case Figure 10 shows a null phase-contrast for all frequencies and Figure 9 no longer shows a peak in phase contrast. It is expected that deeper defects require a higher frequency resolution with a smaller frequency increment $\Delta f$, and consequently longer testing periods. By comparing Figures 9 and 10 it can be concluded that both approaches produce curves which exhibit similar behaviour when the depth of the defect is changed.

The described behaviour is also visible in Figure 11 . For $d=0,5 \mathrm{~cm}$ the visibility of the defect is greater at $t=7,3242 \times 10^{-4}$, after which the defect visibility is greatly reduced. In the same way, for a depth of $2 \mathrm{~cm}$ the defect is most visible for a smaller frequency of $\mathrm{f}=4,8828 \times 10^{-4}$. In Figure 11 it is also clear how the phase-contrast decreases when defect depth is increased.

\section{Conclusions}

In this paper an analytical model to simulate heat transfer in multi-layered systems is presented. An application to a number of case studies is performed to study the effects that changing the thermal properties of the materials and thicknesses of the layers has on phase-contrast results. Phase-contrast is calculated by computing the difference between the phase of heat waves within a system of $m$ layers and a system of $m-1$ layers.

An experimental campaign was carried out using a test specimen with a changeable inclusion/defect. The depth at which the defect is placed is changed, and the resulting phase-contrast curves are compared with those obtained using an analytical model to simulate heat diffusion in multi-layered systems. The generated curves effectively predict the range of frequencies for which the defect is visible.

It is concluded that the heat diffusion simulation using analytical solution for layered systems can be used to contribute to the interpretation of quantitative data results from IRT test performed on building envelopes with defects/inclusions.

\section{Acknowledgements}

The research work presented herein was supported by FEDER funds through the Operational Programme for Competitiveness Factors - Compete and by national funds through the FCT - Portuguese Foundation for Science and Technology, under research project PTDC/ECM/114189/2009 and was supported in part by QREN - Compete under the research project Active Floor Project (FCOMP-01-0202-FEDER-021583. This work has also been supported by the Energy and Mobility for Sustainable Regions - EMSURE - Project (CENTRO-07-0224-FEDER-002004) and the doctoral FCT grant SFRH/BD/91686/2012.

\section{REFERENCES}

[1] Maierhofer Ch, Arndt R, Röllig M. "Influence of concrete properties on the detection of voids with impulsethermography”. Infrared Physics and Technology, vol. 49, pp. 213-217, 2007.

[2] Maierhofer Ch, Wiggenhauser H, Brink A, Röllig M. "Quantitative numerical analysis of transient IR-experiments on buildings". Infrared Physics \& Technology, vol. 46 (1-2), pp. 173-180, 2004.

[3] Balaras C A, Argiriou A A. Infrared thermography for building diagnostics. Energy and Buildings, vol. 34, pp. 171-183, 2002.

[4] Maldague X. "Theory and Practice of Infrared Technology for Non-destructive Testing". John Wiley \& Sons. New York, 2001.

[5] Maldague X. "Introduction to NDT by Active Infrared Thermography". Materials Evaluation, vol. 6 (9), pp. 10601073, 2002.

[6] Maldague X., Marinetti S. "Pulse phase infrared thermography", Applied Physics, vol. 79 (5), pp. 2694-2698, 1996.

[7] Arndt, R.W., "Square pulse thermography in frequency domain as adaptation of pulsed phase thermography for qualitative and quantitative applications in cultural heritage and civil engineering", Infrared Physics \& Technology, vol. 53 (4), pp. 246-253, 2010.

[8] Özisik M.N., "Heat Conduction", second edition, John Wiley \& Sons, New York, 1993.

[9] Haji-Sheikh A., Beck J.V., "Temperature solution in multi-dimensional multi-layer bodies". International Journal of Heat Mass Transfer, vol. 45, pp. 1865-77, 2002.

[10] Tadeu A., Simões N., "Three-dimensional fundamental solutions for transient heat transfer by conduction in an unbounded medium, half-space, slab and layered media”. Engineering Analysis with Boundary Elements, vol. 30 (5), pp. 338-349, 2006.

[11] Tadeu A., António J., Simões N., "2.5D Green's functions in the frequency domain for heat conduction problems in unbounded, half-space, slab and layered media". Computer Modeling in Engineering and Sciences-CMES vol. 6 (1), pp. 43-58, 2004. 